\title{
Challenging 30-day mortality as a site-specific quality metric in non-small cell lung cancer
}

\author{
Carrie B. Moore, MD, PhD, ${ }^{a}$ Morgan L. Cox, MD, ${ }^{a}$ Michael S. Mulvihill, MD, ${ }^{a}$ Jacob Klapper, MD, \\ Thomas A. D’Amico, MD, ${ }^{b}$ and Matthew G. Hartwig, MD, MHS ${ }^{b}$
}

\begin{abstract}
Objective: The objective of this project was to assess the best measure for postoperative outcomes by comparing 30-day and 90-day mortality rates after surgery for non-small cell lung cancer using the National Cancer Database. Secondarily, hospital performance was examined at multiple postoperative intervals to assess changes in ranking based on mortality up to 1 year after surgery.
\end{abstract}

Methods: Patients who had undergone surgery for non-small cell lung cancer between 2004 and 2013 were identified in the National Cancer Database. Mortality rates at 30 days and 90 days were compared after adjusting for several patient characteristics, tumor variables, and hospital procedural volume using generalized logistic mixed models. Subsequently, mixed model logistic regression models were employed to evaluate hospital performance based on calculated mortality at prespecified time points.

Results: A total of 303,579 patients with non-small cell lung cancer were included for analysis. The 90-day mortality was almost double the 30-day mortality $(3.0 \%$ vs $5.7 \%)$. Several patient characteristics, tumor features, and hospital volume were significantly associated with mortality at both 30 days and 90 days. Hospital rankings fluctuate appreciably between early mortality time points, which is additional evidence that quality metrics need to be based on later mortality time points.

Conclusions: Thirty-day mortality is the commonly accepted quality measure for thoracic surgeons; however, hospital rankings may be inaccurate if based on this variable alone. Mortality after 90 days appears to be a threshold after which there is less variability in hospital ranking and should be considered as an alternative quality metric in lung cancer surgery. (J Thorac Cardiovasc Surg 2019;158:570-8)

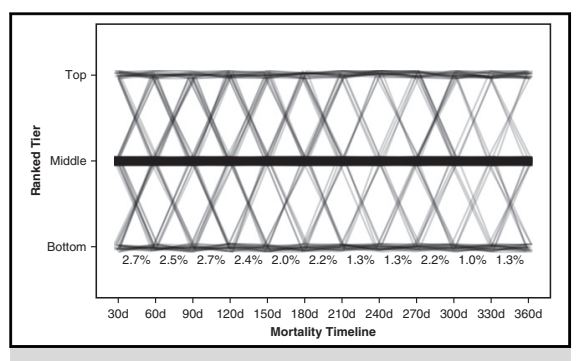

Hospital ranking variation over the course of 1 year.

\section{Central Message}

Mortality estimates after 90 days appear to be a threshold after which there is less variability in hospital ranking and should be considered as an alternative quality metric in lung cancer surgery.

\section{Perspective}

Thirty-day mortality is not the best metric to compare outcomes or facilitate risk-benefit discussions because it underestimates death after lung resection. Evaluation of facility ranking shows $50 \%$ variation in the top facilities by 90 days. Quality improvement projects and ranking algorithms need to incorporate 90-day mortality data to obtain a more accurate understanding of postoperative mortality.

See Commentary on page 579 .
Lung cancer is the most common cause of death by malignancy in the United States and the world. ${ }^{1}$ Despite expanding therapeutic options, the 5-year overall survival for patients with non-small cell lung cancer (NSCLC) remains $<25 \%$. $^{2}$ Surgery is a mainstay of therapy for most patients

From the ${ }^{\mathrm{a} D e p a r t m e n t}$ of Surgery and ${ }^{\mathrm{b}}$ Division of Cardiothoracic Surgery, Duke University Medical Center, Durham, NC.

Funded by the Duke University Hospital Department of Surgery. Dr Mulvihill is supported by the National Heart, Lung, and Blood Institute (No. F32HL132460-02).

Read at the 98th Annual Meeting of The American Association for Thoracic Surgery, San Diego, California, April 28-May 1, 2018.

Received for publication May 1, 2018; revisions received Feb 18, 2019; accepted for publication Feb 22, 2019; available ahead of print May 2, 2019.

Address for reprints: Carrie B. Moore, MD, PhD, Department of Surgery, Duke University Medical Center, 2301 Erwin Rd, Durham, NC 27710 (E-mail: carrie.c. moore@duke.edu).

$0022-5223 / \$ 36.00$

Copyright (c) 2019 by The American Association for Thoracic Surgery

https://doi.org/10.1016/j.jtcvs.2019.02.123 with curable NSCLC. Knowledge of surgery-associated mortality rates and hospital rankings is not only important to improving oncology health care delivery, but also to patients and third-party payers.

Cardiothoracic surgeons have participated in voluntary outcomes reporting for decades in the Society of Thoracic Surgeons (STS) database. ${ }^{3}$ In comparison with national measures, participation in a general thoracic database has been shown to be associated with decreased mortality and

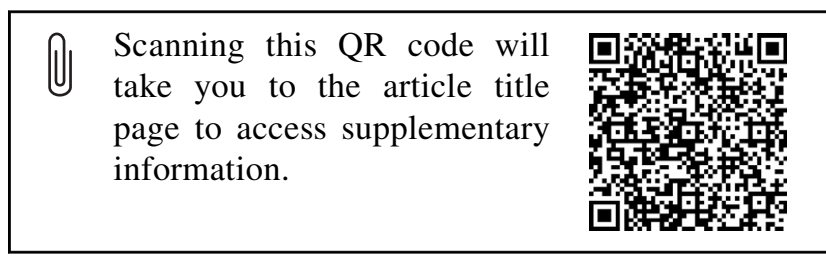




\section{Abbreviations and Acronyms \\ NCDB $=$ National Cancer Database \\ NSCLC $=$ non-small cell lung cancer \\ STS = Society of Thoracic Surgeons}

length of hospitalization. ${ }^{4}$ To ensure highest quality of care and develop plans for improvement, it is critical to measure and compare outcomes at each level of therapy for thoracic oncology patients using national databases.

Thoracic surgeons are often assessed and ranked by 30-day operative mortality, which is unlikely to be the most accurate measure of quality given the complexity of lung cancer resection and risk of complications beyond 30 days. Several studies of patients after pulmonary resection and esophagectomy have indicated that the mortality rate after these operations doubles at 90 days compared with 30-day mortality. ${ }^{5-7}$ Although 30-day mortality was originally introduced to compare cancer centers on immediate morbidity associated with surgical resection, it is likely that longer assessment of outcomes may be important due to the complexity of the operation and longer oncologic survival times. Therefore, the paradigm of accepting 30-day mortality rates as the only measure of quality should continue to be challenged until it is more broadly changed.

In this study, the National Cancer Database (NCDB) was used to examine factors associated with 30-day and 90-day mortality after pulmonary resection for NSCLC. A secondary analysis was performed to compare postoperative mortality across centers using a mixed-model approach at 30-day intervals through 1 year after surgery.

\section{METHODS \\ Data Source}

The NCDB is a joint project of the Commission on Cancer of the American College of Surgeons and the American Cancer Society. Data within NCDB are collected from more than 1500 Commission on Canceraccredited facilities and the NCDB contains more than 34 million historical records. These data can be used to analyze and track patients with malignancies from diagnosis through treatment to outcome. In general, NCDB captures more than $70 \%$ of newly diagnosed cancer cases in the United States. ${ }^{8}$ The 2014 participant user file includes patients diagnosed between 2004 and 2014, and includes survival information for patients diagnosed through 2013.

The data within the NCDB are anonymous; therefore, the study was exempt from institutional review board review and no consent was required. The American College of Surgeons and the Commission on Cancer have not verified and are not responsible for the analytic or statistical methodology employed or the conclusions drawn from these data by investigators.

\section{Study Population}

Patients diagnosed with NSCLC between 2004 and 2013 were identified in the NCDB. Patients were excluded if they did not undergo surgical resection at the reporting facility, had unknown vital status, or were missing values for last contact, days from surgery, or 30-day mortality. To ensure that mortality was directly related to the reporting institution, patients were excluded if the reporting facility did not match the facility where the primary surgery took place. Patients were also excluded if the primary surgical procedure was localized tumor destruction, including laser ablation, cryosurgery, electrocautery, and/or fulguration. Variables such as age, gender, ethnicity, comorbidities (represented as Charlson/Deyo score), insured status, median income quartiles from 2008 to 2012, treatment facility city size, treatment facility type, procedure volume, primary tumor site, analytic stage, histology, grade, neoadjuvant therapy, definitive procedure type, and procedure approach (eg, video-assisted thorascopic surgery, open, or robotic) were obtained from the database. Extent of surgery was document as removal of $<1$ lobe, a lobectomy, pneumonectomy, or not otherwise specified. Pathologic tumor characteristics such as analytic stage and grade were extracted from the NCDB. Stage was reported in accordance with American Joint Committee on Cancer seventh edition. ${ }^{9}$ Histology was extracted and simplified to categories: adenocarcinoma, squamous cell cancer, neuroendocrine, bronchoalveolar cancer, and other as classified in International Classification of Disease for Oncology third revision. ${ }^{10}$ Some patient demographic information such as age and ethnicity were grouped for analytic purposes. Neoadjuvant therapy was defined as any radiation and/or chemotherapy before surgery. Hospital procedure volume was calculated as the procedure volume recorded by a single institution divided by the number of years of data entry provided by that same institution.

Some NCDB variables of interest contained missing data. This is demonstrated by the results of the descriptive analyses (Table 1 and missing data analysis in Tables E1 and E2). Variables with $<5 \%$ missingness were imputed with median values to be included in the regression analyses. Although imputation does introduce a potential source of ambiguity, the benefit is maintaining power and sample size to detect associations. ${ }^{11}$

\section{Statistical Analysis}

Descriptive statistics were calculated for patient characteristics for both 30-day mortality and 90-day mortality. The $\chi^{2}$ test was used to compare categorical variables and the Kruskal-Wallis test was used to compare continuous variables.

Operative mortality rates were stratified into 2 groups: 30 -day mortality and 90-day mortality. Within each group, sequential mixed-effects logistic regression models using a hospital identifier as a random effect were generated under 4 conditions using R (R Foundation for Statistical Computing, Vienna, Austria). Model 1 was built using a hospital identifier as a random effect without any covariates. Model 2 added patient and tumor factors such as demographic characteristics and tumor stage. Model 3 added facility type. Model 4 added procedure volume within specific ranges. Variation between these 4 models was used to calculate mortality attributable to patient and tumor factors (Model 2) and hospital level factors (Models 3 and 4). Model 4 was ultimately used as the final regression model to identify predictors of 30-day and 90-day mortality. Model significance for the odds ratios (ORs) were based on $95 \%$ confidence interval (CI) and the $P$ value.

Subsequently, risk-adjusted mixed effect logistic regression models were used to evaluate hospital performance based on calculated mortality at prespecified time points every 30 days between 30-day mortality and 360-day mortality. Because this analysis was primarily used to assess quality of care at each center, center-level variables, including urban versus rural population size, procedure volume, and facility type, were excluded in this model. ${ }^{12}$ Each hospital was ranked using an Empirical Bayes prediction of center effects for each time point. Similar to STS public reporting ranking system, hospitals were ranked into the highest $(2.5 \%)$, middle $(95 \%)$, and lowest $(2.5 \%)$ performance groups.

\section{RESULTS}

There were 379,773 patients with NSCLC who underwent surgery identified within the NCDB at 1230 facilities available for analysis. After data processing, the analytic 
TABLE 1. Unadjusted 30-day and 90-day mortality descriptive statistics

\begin{tabular}{|c|c|c|c|c|c|c|}
\hline \multirow[b]{2}{*}{ Covariate } & \multicolumn{2}{|c|}{ 30-d Mortality } & \multirow[b]{2}{*}{$P$ value* } & \multicolumn{2}{|c|}{ 90-d Mortality } & \multirow[b]{2}{*}{$P$ value* } \\
\hline & n (\%) & Mortality & & n (\%) & Mortality & \\
\hline \multicolumn{7}{|l|}{ Age $(y)$} \\
\hline $18-49$ & $16,735(5.5)$ & 1.5 & \multirow[t]{5}{*}{$<.0001$} & $16,630(5.5)$ & 3.1 & \multirow[t]{5}{*}{$<.0001$} \\
\hline $50-59$ & $49,958(16.5)$ & 1.7 & & $49,626(16.4)$ & 3.5 & \\
\hline $60-69$ & $101,205(33.3)$ & 2.3 & & $100,648(33.3)$ & 4.5 & \\
\hline $70-79$ & $104,686(34.5$ & 3.7 & & $104,129(34.5)$ & 7 & \\
\hline$>79$ & 30,995 (10.2) & 5.3 & & $30,844(10.2)$ & 9.9 & \\
\hline \multicolumn{7}{|l|}{ Sex } \\
\hline Female & $157,107(51.8)$ & 2.1 & \multirow[t]{2}{*}{$<.0001$} & $156,183(51.7)$ & 4 & \multirow[t]{2}{*}{$<.0001$} \\
\hline Male & $146,472(48.2)$ & 3.9 & & $145,694(48.3)$ & 7.5 & \\
\hline \multicolumn{7}{|l|}{ Race } \\
\hline White & $268,832(88.6)$ & 3 & \multirow[t]{3}{*}{.1223} & $267,362(88.6)$ & 5.7 & \multirow[t]{3}{*}{.0001} \\
\hline Black & $24,919(8.2)$ & 2.8 & & $24,771(8.2)$ & 5.4 & \\
\hline Other & $9828(3.2)$ & 2.7 & & 9744 (3.2) & 4.8 & \\
\hline \multicolumn{7}{|l|}{ Comorbidities } \\
\hline 0 & $158,260(52.1)$ & 2.6 & \multirow[t]{3}{*}{$<.0001$} & $157,295(52.1)$ & 5 & \multirow[t]{3}{*}{$<.0001$} \\
\hline 1 & $105,749(34.8)$ & 3 & & $105,197(34.8)$ & 5.8 & \\
\hline 2 & $39,570(13)$ & 4.3 & & 39,385 (13) & 8 & \\
\hline \multicolumn{7}{|l|}{ Insurance status } \\
\hline Not insured & $5473(1.8)$ & 3 & \multirow[t]{6}{*}{$<.0001$} & $5421(1.8)$ & 5.6 & $<.0001$ \\
\hline Private insurance & $96,636(31.8)$ & 2 & & $96,069(31.8)$ & 3.8 & \\
\hline Medicaid & $12,676(4.2)$ & 2.4 & & $12,585(4.2)$ & 5.2 & \\
\hline Medicare & $180,404(59.4)$ & 3.5 & & $179,473(59.5)$ & 6.7 & \\
\hline Other government & $2627(0.9)$ & 2.2 & & $2604(0.9)$ & 4.9 & \\
\hline Unknown & $5763(1.9)$ & 3.3 & & $5725(1.9)$ & 6.6 & \\
\hline Median income & & & & & & \\
\hline$<\$ 38,000$ & $53,167(17.5)$ & 3.5 & $<.0001$ & $52,870(17.5)$ & 6.6 & $<.0001$ \\
\hline$\$ 38,000-\$ 47,900$ & $71,515(23.6)$ & 3.2 & & $71,110(23.6)$ & 6.1 & \\
\hline$\$ 48,000-\$ 62,9000$ & 80,855 (26.6) & 3 & & $80,411(26.6)$ & 5.8 & \\
\hline$>\$ 63,000$ & $93,204(30.7)$ & 2.3 & & $92,708(30.7)$ & 4.5 & \\
\hline Year of diagnosis & & & & & & \\
\hline 2004 & $27,148(8.9)$ & 3.6 & $<.0001$ & 27,035 (9) & 6.6 & $<.0001$ \\
\hline 2005 & 29,177 (9.6) & 3.3 & & 29,106 (9.6) & 6.3 & \\
\hline 2006 & $29,519(9.7)$ & 3.3 & & $29,437(9.8)$ & 6.3 & \\
\hline 2007 & $30,174(9.9)$ & 3.3 & & $30,081(10)$ & 6.2 & \\
\hline 2008 & $30,914(10.2)$ & 3.1 & & $30,784(10.2)$ & 5.9 & \\
\hline 2009 & $31,200(10.3)$ & 2.9 & & $31,075(10.3)$ & 5.6 & \\
\hline 2010 & $31,124(10.3)$ & 2.7 & & $30,993(10.3)$ & 5.3 & \\
\hline 2011 & $31,546(10.4)$ & 2.6 & & $31,364(10.4)$ & 5.1 & \\
\hline 2012 & $31,415(10.3)$ & 2.6 & & $31,149(10.3)$ & 5.1 & \\
\hline 2013 & $31,362(10.3)$ & 2.4 & & $30,853(10.2)$ & 4.6 & \\
\hline Primary site $\dagger$ & & & & & & \\
\hline C340-Main bronchus & $2778(0.9)$ & 6.3 & $<.0001$ & $2761(0.9)$ & 12.1 & $<.0001$ \\
\hline C341-Upper lobe & $17,4349(57.4)$ & 2.8 & & $17,3407(57.4)$ & 5.3 & \\
\hline C342-Middle lobe & $17,032(5.6)$ & 2 & & $16,916(5.6)$ & 3.9 & \\
\hline C343-Lower lobe & $96,322(31.7)$ & 2.9 & & $95,776(31.7)$ & 5.6 & \\
\hline C348-Overlapping & $5122(1.7)$ & 6.4 & & $5102(1.7)$ & 11.7 & \\
\hline C349—Not otherwise specified & $7976(2.6)$ & 6 & & 7915 (2.6) & 12 & \\
\hline Analytic stage & & & & & & \\
\hline 0 & $1064(0.4)$ & 2.3 & $<.0001$ & $1054(0.3)$ & 3.2 & $<.0001$ \\
\hline I & $18,6354(61.4)$ & 2.2 & & $18,5233(61.4)$ & 3.9 & \\
\hline II & $48,771(16.1)$ & 3.4 & & $48,522(16.1)$ & 6.5 & \\
\hline III & $39,130(12.9)$ & 4.2 & & $38,969(12.9)$ & 8.6 & \\
\hline
\end{tabular}


TABLE 1. Continued

\begin{tabular}{|c|c|c|c|c|c|c|}
\hline \multirow[b]{2}{*}{ Covariate } & \multicolumn{2}{|c|}{ 30-d Mortality } & \multirow[b]{2}{*}{$P$ value* } & \multicolumn{2}{|c|}{ 90-d Mortality } & \multirow[b]{2}{*}{$P$ value } \\
\hline & n (\%) & Mortality & & n (\%) & Mortality & \\
\hline IV & $12,977(4.3)$ & 8 & & $12,907(4.3)$ & 18.4 & \\
\hline Occult & $112(0)$ & 4.5 & & $110(0)$ & 9.1 & \\
\hline Missing & $4(0)$ & 0 & & $3(0)$ & 0 & \\
\hline Unknown & $15,167(5)$ & 3.4 & & $15,079(5)$ & 6.2 & \\
\hline \multicolumn{7}{|l|}{ Histology } \\
\hline 1-Adenocarcinoma & $12,6239(41.6)$ & 2.5 & $<.0001$ & $12,5536(41.6)$ & 4.8 & $<.0001$ \\
\hline $2-\mathrm{SCC}$ & $82,208(27.1)$ & 4.3 & & $81,769(27.1)$ & 8 & \\
\hline 3-Carcinoid & $17,798(5.9)$ & 1.1 & & $17,633(5.8)$ & 1.9 & \\
\hline $4-\mathrm{BAC}$ & $35,380(11.7)$ & 1.8 & & $35,191(11.7)$ & 3.4 & \\
\hline 5-Other & $41,954(13.8)$ & 3.5 & & $41,748(13.8)$ & 7.3 & \\
\hline \multicolumn{7}{|l|}{ Grade } \\
\hline Well diff & $44,314(14.6)$ & 1.9 & $<.0001$ & $44,011(14.6)$ & 3.2 & $<.0001$ \\
\hline Mod diff & $120,998(39.9)$ & 2.7 & & $120,337(39.9)$ & 5 & \\
\hline Poorly diff & $102,434(33.7)$ & 3.6 & & $101,938(33.8)$ & 7.2 & \\
\hline Undiff, anaplastic & $5937(2)$ & 3.9 & & $5894(2)$ & 8.9 & \\
\hline Unknown & $29,896(9.8)$ & 3.3 & & 29,697 (9.8) & 6.3 & \\
\hline \multicolumn{7}{|l|}{ NeoAdj } \\
\hline No & $288,892(95.2)$ & 2.9 & .0017 & $287,268(95.2)$ & 5.6 & $<.0001$ \\
\hline Yes & $14,687(4.8)$ & 3.4 & & $14,609(4.8)$ & 7.4 & \\
\hline \multicolumn{7}{|l|}{ Surgical procedure } \\
\hline$<1$ Lobe removed & $68,695(22.6)$ & 3.2 & $<.0001$ & $68,272(22.6)$ & 6.4 & $<.0001$ \\
\hline Lobectomy & $215,498(71)$ & 2.5 & & $214,314(71)$ & 4.7 & \\
\hline Pneumonectomy & $15,524(5.1)$ & 8.1 & & $15,455(5.1)$ & 13.7 & \\
\hline Not otherwise specified & $3862(1.3)$ & 5.3 & & $3836(1.3)$ & 13 & \\
\hline \multicolumn{7}{|l|}{ Facility type } \\
\hline Community & $20,802(6.9)$ & 4.4 & $<.0001$ & $20,703(6.9)$ & 7.9 & $<.0001$ \\
\hline Comprehensive community & $132,402(43.6)$ & 3.3 & & $131,863(43.7)$ & 6.2 & \\
\hline Academic/research & $115,669(38.1)$ & 2.3 & & $114,837(38)$ & 4.7 & \\
\hline Integrated network & $32,102(10.6)$ & 3 & & $31,891(10.6)$ & 5.8 & \\
\hline \multicolumn{7}{|l|}{ Surgery Approach } \\
\hline Robotic & $7492(2.5)$ & 1.6 & $<.0001$ & $7385(2.4)$ & 3.1 & $<.0001$ \\
\hline VATS & $30,534(10.1)$ & 2 & & $30,280(10)$ & 3.9 & \\
\hline Open (inc conv) & $87,416(28.8)$ & 2.8 & & $86,689(28.7)$ & 5.5 & \\
\hline \multicolumn{7}{|l|}{ Urban vs rural } \\
\hline Metro $>1$ million & $150,193(49.5)$ & 2.7 & $<.0001$ & $149,380(49.5)$ & 5.1 & $<.0001$ \\
\hline Metro 250,000-1 million & $62,102(20.5)$ & 3.1 & & $61,815(20.5)$ & 5.8 & \\
\hline Metro $<250,000$ & $29,461(9.7)$ & 3.3 & & $29,306(9.7)$ & 6.3 & \\
\hline Urban $>20,000$ near metro & $14,384(4.7)$ & 3.4 & & $14,283(4.7)$ & 6.4 & \\
\hline Urban $>20,000$ & $3833(1.3)$ & 3.1 & & $3810(1.3)$ & 5.8 & \\
\hline Urban $2500-20,000$ near metro & $17,728(5.8)$ & 3.3 & & $17,619(5.8)$ & 6.2 & \\
\hline Urban $2500-20,000$ & 8799 (2.9) & 3.6 & & $8730(2.9)$ & 6.4 & \\
\hline Rural $<2500$ near metro & $2766(0.9)$ & 3.4 & & $2747(0.9)$ & 6.3 & \\
\hline Rural $<2500$ & $3209(1.1)$ & 3.6 & & $3185(1.1)$ & 6.8 & \\
\hline \multicolumn{7}{|c|}{ Average annual procedure volume (cases) } \\
\hline $1-3$ & $1627(0.5)$ & 5.5 & $<.0001$ & $1622(0.5)$ & 9 & $<.0001$ \\
\hline $4-9$ & $11,840(3.9)$ & 4 & & $11,787(3.9)$ & 7 & \\
\hline $10-20$ & $45,877(15.1)$ & 3.5 & & $45,647(15.1)$ & 6.6 & \\
\hline$>20$ & $24,4235(80.5)$ & 2.8 & & $24,2821(80.4)$ & 5.4 & \\
\hline \multicolumn{7}{|l|}{ Overall LOS $\S$} \\
\hline Days & $7.127(4-8)$ & & $<.0001$ & $7.128(4-8)$ & & $<.0001$ \\
\hline
\end{tabular}

SCC, Squamous cell cancer; BAC, bronchioloalveolar cancer; Well diff, well differentiated; Mod diff, moderately differentiated; Poorly diff, poorly differentiated; Undiff, anaplastic, undifferentiated, anaplastic; NeoAdj, neoadjuvant; VATS, video-assisted thoracic surgery; Open (inc conv), open, including conversion to open; LOS, length of stay. *Calculated using $\chi^{2}$ analyses and Kruskal-Wallis testing. †Anatomic location of the tumor. The coding is derived from the ICD-O-3 topographical classification. ${ }^{10} \doteqdot$ †Defined as a tumor that overlaps the boundaries of 2 or more subsites and the point of origin is unknown. §Values are presented as median (interquartile range). 
cohort for 30-day and 90-day mortality analyses was 303,579 patients and 301,877 patients, respectively, at 1190 reporting facilities (Figure 1).

\section{Patient Characteristics and Unadjusted Analyses}

At the time point of 30 days, more than $67 \%$ of patients were aged 60 to 79 years, more than $51 \%$ were women, and almost $90 \%$ of patients were white. Few patients underwent neoadjuvant therapy $(4.8 \%)$. The most commonly reported tumor type was adenocarcinoma $(41.6 \%)$ followed by squamous cell carcinoma $(27.1 \%)$. Most patients underwent a lobectomy $(71 \%)$, followed by removal of $<1$ lobe $(22.6 \%)$, and then pneumonectomy $(5.1 \%)$.

Overall, the 90 -day mortality was $5.7 \%$ compared with a 30 -day mortality of $3.0 \%$. In both groups, higher mortality was significantly associated with increasing age, male gender, increasing number of comorbidities, Medicare or uninsured status, lower income, earlier year of diagnosis, primary site of tumor, analytic stage, tumor histology, tumor grade, receipt of neoadjuvant therapy, surgical procedure type, facility type, surgical approach, facility location (city size), facilities that perform fewer than 20 procedures per year, and increased length of stay (Table 1).

\section{Adjusted 30-Day and 90-Day Overall Mortality}

After adjusting for variables in Model 4, 30-day and 90day mortality regression models demonstrated significance for several patient, tumor, and hospital factors (Table 2). Because clinically relevant variables were selected for regression modeling, most were significantly associated with mortality at each time point. For example, across both models, increasing age was associated with increased mortality. Patients older than age 80 years had a 4 times increased risk of dying compared with patients younger than age 50 years. Male gender increased odds of mortality by more than $50 \%$. Compared with patients without insurance, private insurance reduced the risk of death in each mortality group approximately $30 \%$. For each unit increase in median income more than $\$ 38,000$, there was a reduction of mortality odds by almost $10 \%$ in both mortality groups. Squamous cell carcinoma histology was also associated with an almost $40 \%$ increased risk of death in both mortality groups. The biggest reduction in risk was gained when comparing academic facilities to community cancer programs (30-day mortality OR, 0.68; 95\% CI, 0.60-0.077 and 90-day mortality OR, $0.72 ; 95 \% \mathrm{CI}, 0.65-0.80$ ). Compared with facilities that performed 1 to 3 procedures annually, those facilities that performed more than 20 procedures annually were associated with $32 \%$ and $22 \%$ reduction of risk at 30-day and 90-day mortality, respectively. After adjusting for other covariates, differences in neither surrounding population size near a facility nor race were significantly associated with increased mortality.

In addition to the many similarities mentioned above and shown in Table 2, there was a difference between the 2 models. Receipt of neoadjuvant therapy was not significantly associated with mortality in the 30-day mortality model; however, it was significant in the 90-day mortality model. For example, although use of neoadjuvant therapy was not significantly associated with mortality at 30-days, it was associated with a $10 \%$ increased risk of mortality at 90 days (90-day mortality OR, 1.10; 95\% CI, 1.03-1.18).

\section{Comparison of Hospital Rank Using Interval Mortality Time Points}

Risk-adjusted estimates of center rates were used to create hospital rankings at 12 interval mortality time points

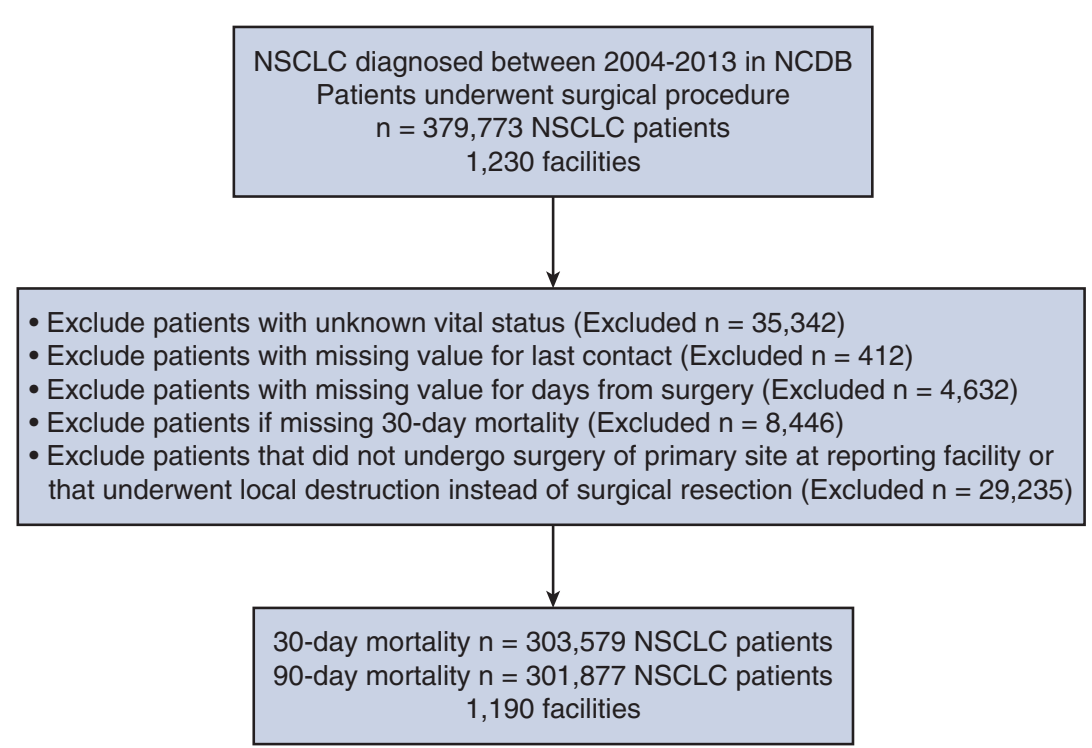

FIGURE 1. Analytic cohort. $N S C L C$, Non-small cell lung cancer; $N C D B$, National Cancer Database. 
TABLE 2. Risk-adjusted 30-day and 90-day mortality results

\begin{tabular}{|c|c|c|c|c|c|c|}
\hline \multirow[b]{2}{*}{ Variable } & \multicolumn{3}{|c|}{ 30-d Mortality } & \multicolumn{3}{|c|}{ 90-d Mortality } \\
\hline & OR & $\mathbf{L L}$ & $\overline{\mathbf{U L}}$ & OR & $\mathbf{L L}$ & UL \\
\hline \multicolumn{7}{|l|}{ Age (y) } \\
\hline $18-49$ & Refere & & & Refer & & \\
\hline $50-59$ & 1.19 & 1.03 & 1.38 & 1.21 & 1.09 & 1.34 \\
\hline $60-69$ & 1.62 & 1.41 & 1.87 & 1.58 & 1.43 & 1.75 \\
\hline $70-79$ & 2.73 & 2.37 & 3.15 & 2.57 & 2.33 & 2.85 \\
\hline $80+$ & 4.24 & 3.67 & 4.92 & 4.09 & 3.68 & 4.55 \\
\hline \multicolumn{7}{|l|}{ Sex } \\
\hline Female & Refere & & & Refer & & \\
\hline Male & 1.54 & 1.47 & 1.61 & 1.58 & 1.52 & 1.63 \\
\hline \multicolumn{7}{|l|}{ Race } \\
\hline White & Refere & & & Refer & & \\
\hline Black & 0.98 & 0.90 & 1.07 & 0.93 & 0.88 & 1.00 \\
\hline Other & 1.09 & 0.95 & 1.24 & 0.99 & 0.89 & 1.09 \\
\hline \multicolumn{7}{|l|}{ Comorbidities } \\
\hline Charlson/Deyo score & 1.21 & 1.17 & 1.24 & 1.20 & 1.17 & 1.23 \\
\hline \multicolumn{7}{|l|}{ Insurance status } \\
\hline Not insured & Refere & & & Refer & & \\
\hline Private insurance & 0.68 & 0.58 & 0.81 & 0.71 & 0.62 & 0.80 \\
\hline Medicaid & 0.82 & 0.67 & 0.99 & 0.95 & 0.82 & 1.10 \\
\hline Medicare & 0.82 & 0.69 & 0.97 & 0.90 & 0.79 & 1.02 \\
\hline Other government insurance & 0.59 & 0.43 & 0.81 & 0.74 & 0.59 & 0.92 \\
\hline Unknown & 1.04 & 0.83 & 1.31 & 1.09 & 0.92 & 1.30 \\
\hline \multicolumn{7}{|l|}{ Income } \\
\hline Median income quartiles & 0.93 & 0.90 & 0.95 & 0.92 & 0.91 & 0.94 \\
\hline \multicolumn{7}{|l|}{ Year of diagnosis } \\
\hline 2004-2013 & 0.97 & 0.96 & 0.98 & $\mathbf{0 . 9 7}$ & 0.97 & 0.98 \\
\hline \multicolumn{7}{|l|}{ Primary site* } \\
\hline C340_-Main bronchus & Refere & & & Refer & & \\
\hline C341-Upper lobe & 0.79 & 0.67 & 0.93 & 0.75 & 0.66 & 0.85 \\
\hline C342-Middle lobe & 0.63 & 0.52 & 0.77 & 0.64 & 0.55 & 0.74 \\
\hline C343-Lower lobe & 0.80 & 0.68 & 0.95 & $\mathbf{0 . 8 0}$ & 0.70 & 0.91 \\
\hline C348-Overlapping & 1.23 & 1.01 & 1.50 & 1.19 & 1.02 & 1.38 \\
\hline C349-Not otherwise specified & 1.12 & 0.93 & 1.36 & 1.09 & 0.95 & 1.26 \\
\hline \multicolumn{7}{|l|}{ Analytic stage } \\
\hline 0 & Refere & & & Refer & & \\
\hline I & 0.89 & 0.59 & 1.35 & 1.15 & 0.82 & 1.61 \\
\hline II & 1.21 & 0.80 & 1.83 & 1.73 & 1.24 & 2.43 \\
\hline III & 1.53 & 1.01 & 2.32 & 2.38 & 1.70 & 3.33 \\
\hline IV & 3.29 & 2.16 & 5.00 & 6.11 & 4.36 & 8.57 \\
\hline Occult & 1.55 & 0.57 & 4.23 & 2.33 & 1.11 & 4.92 \\
\hline Unknown & 1.75 & 1.14 & 2.67 & 2.33 & 1.65 & 3.29 \\
\hline \multicolumn{7}{|l|}{ Histology } \\
\hline 1-Adenocarcinoma & Refere & & & Refer & & \\
\hline $2-\mathrm{SCC}$ & 1.38 & 1.31 & 1.45 & 1.44 & 1.38 & 1.50 \\
\hline 3-Carcinoid & 0.46 & 0.39 & 0.54 & 0.43 & 0.38 & 0.48 \\
\hline $4-\mathrm{BAC}$ & 0.83 & 0.76 & 0.91 & 0.82 & 0.76 & 0.87 \\
\hline 5-Other & 1.17 & 1.09 & 1.25 & 1.33 & 1.27 & 1.40 \\
\hline \multicolumn{7}{|l|}{ Grade } \\
\hline 1-4, unknown & 1.04 & 1.03 & 1.05 & 1.04 & 1.03 & 1.05 \\
\hline \multicolumn{7}{|l|}{ Neoadjuvant therapy } \\
\hline No & Refere & & & Refer & & \\
\hline Yes & 1.01 & 0.91 & 1.11 & 1.10 & 1.03 & 1.18 \\
\hline
\end{tabular}


TABLE 2. Continued

\begin{tabular}{|c|c|c|c|c|c|c|}
\hline \multirow[b]{2}{*}{ Variable } & \multicolumn{3}{|c|}{ 30-d Mortality } & \multicolumn{3}{|c|}{ 90-d Mortality } \\
\hline & $\overline{\text { OR }}$ & $\mathbf{L L}$ & $\mathbf{U L}$ & $\overline{\mathbf{O R}}$ & $\mathbf{L L}$ & UL \\
\hline \multicolumn{7}{|l|}{ Facility type } \\
\hline Community & Refer & & & Refe & & \\
\hline Comprehensive community & 0.87 & 0.78 & 0.97 & $\mathbf{0 . 8 8}$ & $\mathbf{0 . 8 0}$ & 0.96 \\
\hline Academic/research & 0.68 & 0.60 & 0.77 & 0.72 & 0.65 & 0.80 \\
\hline Integrated network & $\mathbf{0 . 8 0}$ & 0.69 & 0.94 & $\mathbf{0 . 8 3}$ & 0.72 & 0.94 \\
\hline \multicolumn{7}{|l|}{ Surgical procedure } \\
\hline$<1$ Lobe removed & Refer & & & Refe & & \\
\hline Lobectomy & 0.91 & 0.86 & 0.96 & 0.88 & 0.85 & 0.92 \\
\hline Pneumonectomy & $\mathbf{2 . 5 0}$ & 2.30 & 2.71 & 2.08 & 1.96 & 2.22 \\
\hline Not otherwise specified & 1.21 & 1.02 & 1.43 & 1.49 & 1.32 & 1.68 \\
\hline \multicolumn{7}{|c|}{ Average annual procedure volume (cases) } \\
\hline $1-3$ & Refer & & & Refe & & \\
\hline $4-9$ & 0.79 & 0.61 & 1.02 & 0.85 & 0.69 & 1.04 \\
\hline $10-20$ & 0.75 & 0.58 & 0.96 & 0.86 & 0.70 & 1.06 \\
\hline $20+$ & 0.68 & 0.53 & $\mathbf{0 . 8 8}$ & 0.77 & 0.63 & 0.95 \\
\hline \multicolumn{7}{|l|}{ Facility city size } \\
\hline Urban/rural & 0.99 & 0.98 & 1.01 & 0.98 & 0.97 & 0.99 \\
\hline
\end{tabular}

Values are presented as odds ratio (OR) and upper limit (UL) and lower limit (LL) of $95 \%$ confidence interval. Generalized logistic model with mixed effects were used to generate risk-adjusted models for 30-day mortality and 90-day mortality. Boldface type indicates statistical significance. SCC, Squamous cell cancer; BAC, bronchioloalveolar cancer. *Anatomic location of the tumor. The coding is derived from the ICD-O-3 topographical classification. ${ }^{10}$

(30 days, 60 days, 90 days, 120 days, 150 days, 180 days, 210 days, 240 days, 270 days, 300 days, 330 days, and 360 days). Table 3 shows the changes in ranking using only 30-day and 90-day mortality. Eleven out of 30 facilities were in the top $2.5 \%$ at 30 days and moved to the middle $95 \%$ at 90 days. Similarly, 12 out of 30 facilities that were in the bottom ranking group at 30 days moved up into the middle ranking group at 90 days. To investigate the trend across all interval mortality points, Figure 2 shows the ranking changes between time points. Line density corresponds to the number of facilities changing ranking groups. The total percentage of facilities changing groups is indicated in text at the bottom of Figure 2. The largest changes were seen between 30-day to 120-day mortality models $(2.5 \%-2.7 \%)$.

\section{DISCUSSION}

Thirty-day mortality is the time point used most often to compare surgical outcomes. In other single-institution

TABLE 3. Number of hospitals that changed ranking categories (bottom $2.5 \%$, middle $95 \%$, or top $2.5 \%$ ) according to 3-tier rating system between 30-day and 90-day mortality

\begin{tabular}{lrrrr}
\hline & \multicolumn{3}{c}{ 90-d Mortality } & \\
\cline { 2 - 4 } & Bottom 2.5\% & Middle 95\% & Top 2.5\% & Total \\
\hline 30-d Mortality & & & & \\
Bottom 2.5\% & 18 & 12 & 0 & 30 \\
Middle 95\% & 12 & 1107 & 11 & 1130 \\
Top 2.5\% & 0 & 11 & 19 & 30 \\
Total & 30 & 1130 & 30 & 1190 \\
\hline
\end{tabular}

studies or smaller database investigations of lung cancer resection, the mortality rate doubles between 30 days and 90 days. ${ }^{5-7}$ In this study, 9007 patients died before the 30day mortality time point. Between 30 days and 90 days, 8097 patients died. This is reflected in the mortality rate, which was almost $3.0 \%$ at 30 days and increased to $5.7 \%$ at 90 days. Similar factors were associated with death at 30 days and 90 days and included patient, tumor, and facility characteristics. It is likely that each mortality time point represents a different concept in patient care. Thirty-day mortality is reported largely to assess immediate perioperative outcomes and safety. However, given advances in critical care, 60-day or 90-day mortality measurements may be a more appropriate measure of postoperative survival and effectiveness of overall oncologic care.

Another important reason to uncover the true estimate of mortality is to appropriately counsel patients. Using only a 30-day mortality estimate would severely underestimate their true risk of death following an operative resection. Others studying colorectal, lung, and esophagus resections have suggested the implementation of 90-day postoperative mortality outcomes for outcome comparison, quality improvement, and patient mortality risk discussions. ${ }^{7,13,14}$

A final aim of this project was to measure the degree of per-center variability in ranking when alternative postoperative mortality rates were employed. The STS star ranking system ranks institutions using outcomes and clinical data to distinguish the top-performing 3-star hospitals (mortality/morbidity rates 2 standard deviations higher than the STS mean), from 2-star hospitals (middle 95\%), and lower-performing 1-star hospitals (mortality/morbidity 


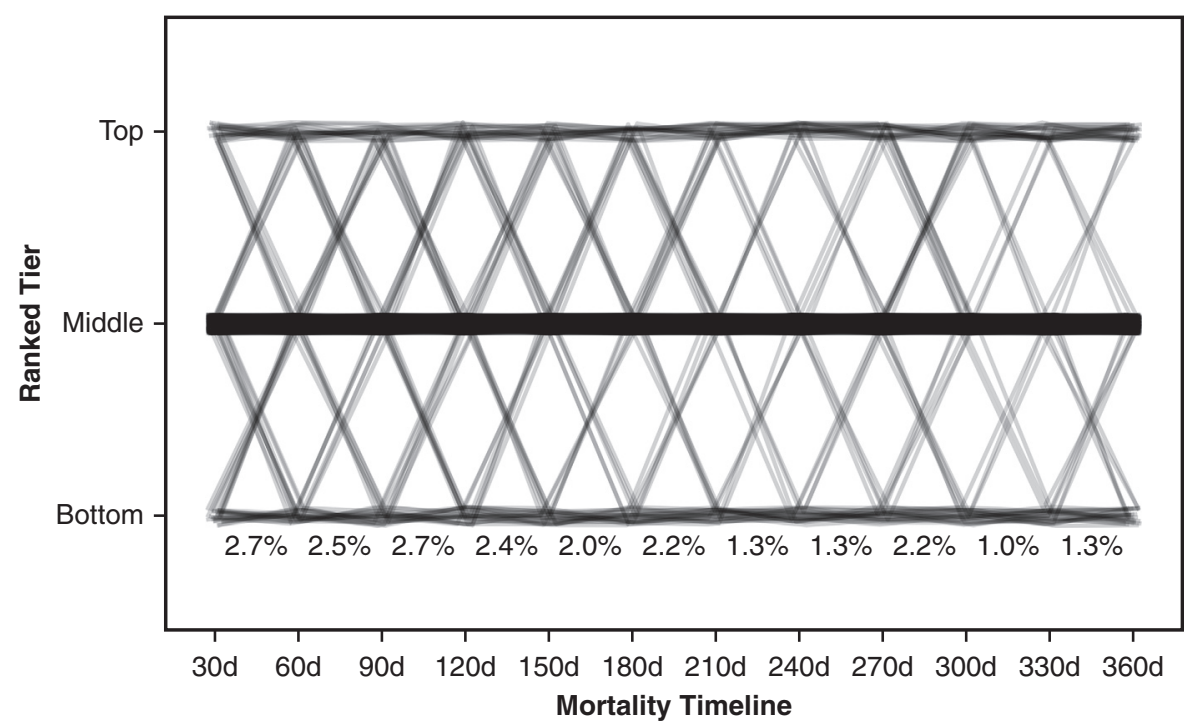

FIGURE 2. Line graph indicating how facilities change ranking groups over 12 interval mortality time points. Density corresponds to the number of facilities changing groups. Values below the graph indicate the percent of facilities that changed ranking groups between time points. For example, $2.8 \%$ of facilities changed ranking groups between 30 days and 60 days.

rates 2 standard deviations lower than the STS mean). Loosely based on that format, in this model, highperforming hospitals were distinguished from middle- and lower-performing hospitals at multiple time points using mortality. As shown in Table 3, the top echelon of facilities (ie, top $2.5 \%$ ) change by almost $50 \%$ between 30 and 90 days. Figure 2 shows the percent changes between time intervals. The maximum fluctuation between ranking groups is between 30 days and 60 days and 90 days and 120 days. One explanation for this is that 30-day and 90day mortality are commonly assessed time points. Therefore, immediately after those dates, patients may be less likely to follow-up or may even transition their care to another facility for further treatment. The changes seem to stabilize after 120 days. The high degree of change in the first 60 days in concerning and could potentially lead to inaccurate comparisons if later mortality figures are ignored. In addition, lower mortality $(<3 \%)$ at 30 days makes it difficult to stratify programs for quality improvement. If only a single mortality time point is to be used, likely 90-day mortality would be more accurate to compare rankings between hospitals.

\section{Study Strengths and Limitations}

The NCDB is among the largest cancer registries in the world and is very useful for studying outcomes in general populations. This investigation using NCDB data is the most comprehensive review of NSCLC surgical mortality at multiple time points to date. Patients with missing vital status or last known contact information had to be excluded from this study. Although our mortality estimates align with published mortality rates, missing data can represent a potential source of bias. Additionally, many patient- and treatment-related factors are not captured in the NCDB. For example, a summative Charlson/Deyo score is provided, but additional details regarding the severity of comorbidities are not available. There is also limited information available in the NCDB regarding in-hospital postoperative course. For example, there are no data detailing major postoperative morbidities, reoperation for oncologic resection, reoperation to address postoperative complications, readmission to hospitals other than the index hospital, and/or transfers between facilities. More granular data for comorbidities, postoperative course, and detailed cancer therapy would improve our ability to accurately adjust for these factors and improve the quality of models generated from the NCDB. ${ }^{15}$

\section{CONCLUSIONS}

This study echoes concern that 30-day mortality may not the most appropriate metric to compare outcomes, to facilitate risk-benefit discussions with patients, or to use as the sole variable in a ranking system to distinguish institutions of excellence. This study provides further evidence that 30day mortality underestimates the incidence of death following lung resection for NSCLC, which doubles by 90 days. Evaluation of hospital performance shows as quantifiable fluctuation in the top tier facilities between 30 and 90 days. Quality improvement projects and ranking algorithms should consider incorporation of 90-day mortality data to obtain a more accurate understanding of postoperative mortality. 


\section{Conflict of Interest Statement}

Authors have nothing to disclose with regard to commercial support.

\section{References}

1. US Cancer Statistics. Leading cancer cases and deaths, male and female, 2015. Available at: https://nccd.cdc.gov/uscs/cancersbyraceandethnicity.aspx. Accessed April 5, 2018.

2. National Cancer Institute Surveillance, Epidemiology, and End Results Program. Table 15.14. Non-small cell cancer of the lung and bronchus (invasive): 5-year relative and period survival by race, sex, diagnosis year, age and stage at diagnosis. Available at: https://seer.cancer.gov/csr/1975_2014/browse_csr.php? sectionSEL=15\&pageSEL=sect_15_table.14.html. Accessed April 5, 2018.

3. STS Public Reporting Online. General surgery public reporting. Available at: https://publicreporting.sts.org/gtsd. Accessed April 4, 2018.

4. LaPar DJ, Bhamidipati CM, Lau CL, Jones DR, Kozower BD. The Society of Thoracic Surgeons general thoracic surgery database: establishing generalizability to national lung cancer resection outcomes. Ann Thorac Surg. 2012;94:216-21.

5. McMillan RR, Berger A, Sima CS, Lou F, Dycoco J, Rusch V, et al. Thirty-day mortality underestimates the risk of early death after major resections for thoracic malignancies. Ann Thorac Surg. 2014;98:1769-74.

6. Bryant AS, Rudemiller K, Cerfolio RJ. The 30- versus 90-day operative mortality after pulmonary resection. Ann Thorac Surg. 2010;89:1717-22.

7. In H, Palis BE, Merkow RP, Posner MC, Ferguson MK, Winchester DP, et al. Doubling of 30-day mortality by 90 days after esophagectomy: a crit- ical measure of outcomes for quality improvement. Ann Surg. 2016;263: 286-91.

8. American College of Surgeons National Cancer Database. Available at: https:// www.facs.org/quality-programs/cancer/ncdb. Accessed April 3, 2018

9. Edge S, Byrd DR, Compton CC, Fritz AG, Greene F, Trotti A, eds. AJCC Cancer Staging Handbook. New York: Springer-Verlag; 2010.

10. International Classification of Diseases for Oncology. ICD-O-3 Online. Available at: http://codes.iarc.fr/. Accessed April 4, 2018.

11. Pedersen AB, Mikkelsen EM, Cronin-Fenton D, Kristensen NR, Pham TM, Pedersen L, et al. Missing data and multiple imputation in clinical epidemiological research. Clin Epidemiol. 2017;9:157-66.

12. MacKenzie TA, Grunkemeier GL, Grunwald GK, O’Malley AJ, Bohn C, Wu Y, et al. A primer on using shrinkage to compare in-hospital mortality between centers. Ann Thorac Surg. 2015;99:757-61.

13. Visser BC, Keegan H, Martin M, Wren SM. Death after colectomy: it's later than we think. Arch Surg. 2009;144:1021-7.

14. Hu Y, McMurry TL, Wells KM, Isbell JM, Stunkeborg GJ, Kozower BD. Postoperative mortality is an inadequate quality indicator for lung cancer resection. Ann Thorac Surg. 2014;97:973-9.

15. Boff DJ, Rosen JE, Mallin K, Loomis A, Gay G, Palis B, et al. Using the national cancer database for outcomes research: a review. JAMA Oncol. 2017;3:1722-8.

Key Words: 30-day mortality, 90-day mortality, quality metrics, hospital ranking, lung cancer surgery, National Cancer Database (NCDB) 
TABLE E1. Three variables with missingness were imputed. The percent missing in each of those variables are shown

\begin{tabular}{lc}
\hline & Number missing $(\%)$ \\
\hline Facility type & $2604(1.0)$ \\
Median income & $4838(1.6)$ \\
Urban vs rural & $11,104(3.7)$ \\
\hline
\end{tabular}


TABLE E2. Comparison of 30-day mortality generalized logistic model using completed imputed data set compared with the original data set where patients with missingness in facility type, median income, and urban versus rural location variables are dropped

\begin{tabular}{|c|c|c|c|c|c|c|}
\hline \multirow[b]{2}{*}{ Variable } & \multicolumn{3}{|c|}{ 30-d Mortality imputed } & \multicolumn{3}{|c|}{ 30-d Mortality without imputation } \\
\hline & OR & $\mathbf{L L}$ & UL & OR & $\mathbf{L L}$ & $\mathbf{U L}$ \\
\hline \multicolumn{7}{|l|}{ Age $(y)$} \\
\hline $18-49$ & Reference & & & Reference & & \\
\hline $50-59$ & 1.19 & 1.03 & 1.38 & 1.12 & 0.96 & 1.31 \\
\hline $60-69$ & 1.62 & 1.41 & 1.87 & 1.53 & 1.32 & 1.77 \\
\hline $70-79$ & 2.73 & 2.37 & 3.15 & 2.57 & 2.21 & 2.99 \\
\hline $80+$ & 4.24 & 3.67 & 4.92 & 4.02 & 3.43 & 4.71 \\
\hline \multicolumn{7}{|l|}{ Sex } \\
\hline Female & Reference & & & Reference & & \\
\hline Male & 1.54 & 1.47 & 1.61 & 1.55 & 1.48 & 1.62 \\
\hline \multicolumn{7}{|l|}{ Race } \\
\hline White & Reference & & & Reference & & \\
\hline Black & 0.98 & 0.90 & 1.07 & 0.98 & 0.90 & 1.07 \\
\hline Other & 1.09 & 0.95 & 1.24 & 1.06 & 0.93 & 1.21 \\
\hline \multicolumn{7}{|l|}{ Comorbidities } \\
\hline Charlson/Deyo/score & 1.21 & 1.17 & 1.24 & 1.21 & 1.17 & 1.25 \\
\hline \multicolumn{7}{|l|}{ Insurance status } \\
\hline Not insured & Reference & & & Reference & & \\
\hline Private insurance & 0.68 & 0.58 & 0.81 & 0.69 & 0.58 & 0.82 \\
\hline Medicaid & 0.82 & 0.67 & 0.99 & 0.84 & 0.68 & 1.02 \\
\hline Medicare & 0.82 & 0.69 & 0.97 & 0.84 & 0.70 & 1.00 \\
\hline Other government insurance & 0.59 & 0.43 & 0.81 & 0.59 & 0.42 & 0.82 \\
\hline Unknown & 1.04 & 0.83 & 1.31 & 1.02 & 0.80 & 1.30 \\
\hline \multicolumn{7}{|l|}{ Income } \\
\hline Median income quartiles & 0.93 & 0.90 & 0.95 & 0.92 & 0.90 & 0.95 \\
\hline \multicolumn{7}{|l|}{ Year of diagnosis } \\
\hline 2004-2013 & 0.97 & 0.96 & 0.98 & 0.97 & 0.96 & 0.98 \\
\hline \multicolumn{7}{|l|}{ Primary site* } \\
\hline C340-Main bronchus & Reference & & & Reference & & \\
\hline C341-Upper lobe & 0.79 & 0.67 & 0.93 & 0.77 & 0.65 & 0.91 \\
\hline C342-Middle lobe & 0.63 & 0.52 & 0.77 & 0.60 & 0.49 & 0.74 \\
\hline C343-Lower lobe & 0.80 & 0.68 & 0.95 & 0.78 & 0.66 & 0.93 \\
\hline C348-Overlapping & 1.23 & 1.01 & 1.50 & 1.20 & 0.98 & 1.47 \\
\hline C349-Not otherwise specified & 1.12 & 0.93 & 1.36 & 1.11 & 0.91 & 1.34 \\
\hline \multicolumn{7}{|l|}{ Analytic stage } \\
\hline 0 & Reference & & & Reference & & \\
\hline I & 0.89 & 0.59 & 1.35 & 0.94 & 0.61 & 1.45 \\
\hline II & 1.21 & 0.80 & 1.83 & 1.26 & 0.82 & 1.95 \\
\hline III & 1.53 & 1.01 & 2.32 & 1.60 & 1.04 & 2.47 \\
\hline IV & 3.29 & 2.16 & 5.00 & 3.49 & 2.25 & 5.39 \\
\hline Occult & 1.55 & 0.57 & 4.23 & 1.69 & 0.61 & 4.66 \\
\hline Unknown & 1.75 & 1.14 & 2.67 & 1.82 & 1.17 & 2.84 \\
\hline \multicolumn{7}{|l|}{ Histology } \\
\hline 1-Adenocarcinoma & Reference & & & Reference & & \\
\hline $2-\mathrm{SCC}$ & 1.38 & 1.31 & 1.45 & 1.38 & 1.31 & 1.45 \\
\hline 3-Carcinoid & 0.46 & 0.39 & 0.54 & 0.48 & 0.40 & 0.56 \\
\hline 4-BAC & 0.83 & 0.76 & 0.91 & 0.83 & 0.76 & 0.91 \\
\hline 5-Other & 1.17 & 1.09 & 1.25 & 1.18 & 1.11 & 1.27 \\
\hline \multicolumn{7}{|l|}{ Grade } \\
\hline 1-4, unknown & 1.04 & 1.03 & 1.05 & 1.04 & 1.03 & 1.05 \\
\hline
\end{tabular}


TABLE E2. Continued

\begin{tabular}{|c|c|c|c|c|c|c|}
\hline \multirow[b]{2}{*}{ Variable } & \multicolumn{3}{|c|}{ 30-d Mortality imputed } & \multicolumn{3}{|c|}{ 30-d Mortality without imputation } \\
\hline & $\overline{\text { OR }}$ & $\mathbf{L L}$ & $\mathbf{U L}$ & $\overline{\text { OR }}$ & $\mathbf{L L}$ & $\mathbf{U L}$ \\
\hline \multicolumn{7}{|l|}{ Neoadjuvant therapy } \\
\hline No & Reference & & & Reference & & \\
\hline Yes & 1.01 & 0.91 & 1.11 & 1.01 & 0.91 & 1.12 \\
\hline \multicolumn{7}{|l|}{ Facility type } \\
\hline Community & Reference & & & Reference & & \\
\hline Comprehensive community & 0.87 & 0.78 & 0.97 & 0.89 & 0.79 & 0.99 \\
\hline Academic/research & 0.68 & 0.60 & 0.77 & 0.69 & 0.61 & 0.79 \\
\hline Integrated network & 0.80 & 0.69 & 0.94 & 0.81 & 0.69 & 0.96 \\
\hline \multicolumn{7}{|l|}{ Surgical procedure } \\
\hline$<1$ Lobe removed & Reference & & & Reference & & \\
\hline Lobectomy & 0.91 & 0.86 & 0.96 & 0.90 & 0.86 & 0.96 \\
\hline Pneumonectomy & 2.50 & 2.30 & 2.71 & 2.48 & 2.28 & 2.70 \\
\hline Not otherwise specified & 1.21 & 1.02 & 1.43 & 1.18 & 0.99 & 1.40 \\
\hline \multicolumn{7}{|c|}{ Average annual procedure volume (cases) } \\
\hline $1-3$ & Reference & & & Reference & & \\
\hline $4-9$ & 0.79 & 0.61 & 1.02 & 0.76 & 0.59 & 0.98 \\
\hline $10-20$ & 0.75 & 0.58 & 0.96 & 0.71 & 0.56 & 0.92 \\
\hline $20+$ & 0.68 & 0.53 & 0.88 & 0.64 & 0.49 & 0.83 \\
\hline \multicolumn{7}{|l|}{ Facility city size } \\
\hline Urban vs rural & 0.99 & 0.98 & 1.01 & 1.00 & 0.98 & 1.01 \\
\hline
\end{tabular}

Values are presented as odds ratio (OR) and upper limit (UL) and lower limit (LL) of $95 \%$ confidence interval. SCC, Squamous cell cancer; $B A C$, bronchioloalveolar cancer.

*Anatomic location of the tumor. The coding is derived from the ICD-O-3 topographical classification. ${ }^{10}$ 